\title{
«Solche wie Sie wollen wir hier nicht» Homosexuelle in der psychoanalytischen Ausbildung - Rückblick einer Berliner Psychoanalytikerin \\ Julia Braun (Zürich)
}

Zusammenfassung: Der Artikel zeigt auf, in welches Dilemma Homosexuelle bis vor nicht allzu langer Zeit noch gerieten, wenn sie eine psychoanalytische Ausbzw. Weiterbildung machen wollten. Einige Auswirkungen der institutionellen Diskriminierung auf die Situation der AusbildungskandidatInnen, beispielsweise die Notwendigkeit einer Selbstverleugnung als Eintrittskarte in ein psychoanalytisches Institut oder das Verunmöglichen des freien Assoziierens in der Lehranalyse, werden anhand eines Beispiels aus den 1980er Jahren in Deutschland veranschaulicht. Angela Hennig, die ihre psychoanalytische Ausbildung in der Zeit von 1981-1991 in Berlin absolvierte, hat sich dankenswerterweise für eine Berichterstattung zu dem Thema zur Verfügung gestellt.

Schlüsselwörter: Institutionelle Diskriminierung, lesbische Psychoanalytikerin, homosexueller Psychoanalytiker, freie Assoziation, Selbstverleugnung

\section{Einleitung}

Seit Jahren gibt es Erzählungen darüber, dass Homosexuelle an vielen psychoanalytischen Instituten nicht zur Weiterbildung zugelassen werden. Offiziell werde dieses Vorgehen jedoch nicht mit der sexuellen Orientierung der BewerberInnen begründet. Durch die Angabe vorgeschobener Gründe wären die Institute in der Lage, sich von einer Pathologisierung der Homosexualität zu distanzieren, ohne sich einer offenen Diskussion über die Thematik aussetzen zu müssen. Ausgerechnet an den Orten, wo Aufdeckung zur Grundlage der Arbeit gehört, nämlich an psychoanalytischen Instituten, würde so zu- statt aufgedeckt werden. In Folge dieser Situation gab es offenbar Homosexuelle, die ihre psychoanalytische Weiterbildung absolvierten, ohne ihre wahre sexuelle Orientierung preiszugeben - auch in der Lehranalyse nicht. Andere seien auf Institute ausgewichen, an denen sie trotz ihrer homosexuellen Orientierung zugelassen wurden. Der Rede nach seien das jedoch eher Institute gewesen, die nicht von der Internationalen 
Psychoanalytischen Vereinigung (IPV) anerkannt waren. Was ist nun dran an diesen Erzählungen? Wie kann man sich eine solche Diskriminierungssituation vorstellen? Wie sind die Haltungen an den Instituten heute und welche Haltung wäre wünschenswert? Das sind Fragen, die sich aufdrängen und die schwer zu beantworten sind, so lange kein/e Ausbildungskanditat/in wagt, seine/ihre Erfahrungen öffentlich zu machen.

Auch heute, in einer Zeit, in der die gesellschaftliche Akzeptanz von Homosexualität im Vergleich zu den frühen 1980er Jahren merklich gestiegen ist, ist die Sichtbarkeit homosexueller PsychoanalytikerInnen gering und selten auf den ersten Blick gegeben. Sehr bekannte PsychoanalytikerInnen, von deren Homosexualität man weiss, schreiben psychoanalytische Lehrbücher, leiten Tagungen und entwickeln psychoanalytische Theorien. Dass sie homosexuell sind, erfährt man meistens «unter der Hand». Googelt man «lesbische Psychoanalytikerin» im Internet, findet man einen Link zu einem pornografischen Video.

Mein Anliegen ist es, die schwierige Situation, wie nicht-heterosexuelle AusbildungsinteressentInnen und -kandidatInnen sie an vielen psychoanalytischen Ausbildungsinstituten über Jahrzehnte vorfanden, sichtbar zu machen und damit ein breiteres Bewusstsein für diese Form der institutionellen Diskriminierung ${ }^{1} \mathrm{zu}$ schaffen. Auf diese Art erhoffe ich mir, einen Einblick in das Dilemma zu ermöglichen, in dem sich Homosexuelle befanden, wenn sie eine psychoanalytische Ausbildung absolvieren wollten. Der Artikel soll im besten Fall dazu beitragen, dass der Umgang mit Minoritäten an psychoanalytischen Ausbildungsinstituten reflektiert wird, um einen Boden für Veränderungen zu schaffen. Zukünftige InteressentInnen sollen Ausbildungsbedingungen vorfinden können, die weniger Leid verursachen und den KandidatInnen in ihren Lehranalysen ein tatsächlich freies Assoziieren erlauben. Die Lehranalyse darf nicht eingeschränkt sein durch von äusseren Bedingungen konstituierte Ängste vor dem Verstossen-werden, wenn die Analysanden «das Falsche» assoziieren.

\section{Politischer und institutioneller Hintergrund}

In den frühen 1980er Jahren existierte in der Bundesrepublik Deutschland der Paragraf 175 des deutschen Strafgesetzbuches ( $\$ 175$ StGB) seit bereits 110 Jahren. Dieser Paragraf stellte sexuelle Handlungen zwischen Personen männlichen Geschlechts unter Strafe. Dementsprechend existierten begründete Ängste unter homosexuellen Menschen, sich in der Öffentlichkeit zu zeigen. Die Abschaffung des Paragrafen erfolgte erst 1994. 
Als Beginn der Schwulenbewegung gilt in Deutschland die Uraufführung des Films Nicht der Homosexuelle ist pervers, sondern die Situation, in der er lebt (BRD 1970, Regie: Rosa von Praunheim, Text: Martin Dannecker) bei den Berliner Filmfestspielen 1971. Der Film führte Anfang 1972 zur Gründung der Frauengruppe der «Homosexuellen AktionWest-Berlin (HAW)», deren Teilnehmerinnen eine offensive Strategie des Sichtbarwerdens verfolgten. Die Lesbenbewegung verschmolz in den 1970er Jahren zu einem Grossteil mit der Frauenbewegung und prägte dort das Paradigma des Lesbischen Feminismus. Die 1980er Jahre waren gekennzeichnet von einer Institutionalisierung der Lesben- und Schwulenbewegung, in der sich zahlreiche Emanzipationsgruppen bildeten. $^{2}$

Auch in den psychotherapeutischen Bereich hielt diese Entwicklung Einzug. Zwischen 1977 und 2000 wurde jährlich der «Feministische Frauentherapiekongress (FTK)» organisiert, auf dem Leitlinien frauenspezifischer Herangehensweise in der Psychotherapie entwickelt, diskutiert und publiziert wurden. Feministisches Gedankengut floss zunehmend in psychologisch-psychotherapeutisches Handeln ein. Den theoretischen Hintergrund lieferten v. a. die Publikationen von Analytikerinnen, verwoben mit Forderungen aus dem Gleichheits- und Differenzdiskurs. In den 1980er Jahren begann der Aufbau von feministischen Beratungsstellen, Therapie- und Frauengesundheitszentren sowie Aus- und Fortbildungen in feministischer Tradition (vgl. Schigl, 2012).

Der Begründer der Psychoanalyse formulierte eine richtungweisende Sexualtheorie, die von einer polymorph-perversen infantilen Sexualität ausgeht. Nach ihr ist Heterosexualität ebenso erklärungsbedürftig wie Homosexualität (Freud, 1905). Zweifellos war Freud, mitsamt der Widersprüche und Selbstkorrekturen in seinen Schriften, wesentlich neugieriger und offener als die meisten seiner Nachfolger. In seinem Werk gab es zwar normative Annahmen bezüglich des Geschlechtslebens, andererseits erklärte er explizit,

... dass Homosexuelle nicht unbedingt psychisch labiler seien als Heterosexuelle, dass Homosexuelle in der Tat als Analytiker dienen könnten und dass es so wenig Aussicht auf die Konversion Homosexueller zur Heterosexualität gebe wie das Gegenteil. (Herzog, 2015, S. 417)

Bereits 1905 schrieb Freud in den «Drei Abhandlungen zur Sexualtheorie» trotz der damals allgemein verbreiteten Pathologisierung der homosexuellen Orientierung in den Humanwissenschaften: «Die psychoanalytische Forschung 
widersetzt sich mit aller Entschiedenheit dem Versuch, die Homosexuellen als eine besonders geartete Gruppe von den anderen Menschen abzutrennen» (1905, S. 44). Und 1921 widersprach er seinem Kollegen Ernest Jones, der einem homosexuellen Arzt die Zulassung zur psychoanalytischen Ausbildung verwehrt hatte.

Im Zuge der Institutionalisierung der Psychoanalyse aber kam es bei den Schülern Freuds zu einer ideologischen Einengung der freudschen Theorien (vgl. Kreische, 2005, S. 120). So stand die Psychoanalyse ganz entgegen ihrem emanzipatorischen Selbstverständnis über Jahrzehnte unter dem Einfluss einer Pathologisierung der Homosexualität. Diese Haltung führte zu einer grundsätzlichen Ablehnung von schwulen und lesbischen BewerberInnen für die psychoanalytische Ausbildung. «Wollten diese dennoch die Ausbildung absolvieren, mussten sie das incognito tun - eine leidvolle Variante, die oft das ganze weitere Berufsleben überschattete» (Homepage des DPG-Instituts Hamburg). Erst in den 1970er Jahren fand eine Ablösung durch eine konstruktive Weiterentwicklung der Theorie statt, die bis in die Gegenwart anhält (vgl. Kreische, 2005, S. 120).

In den USA waren vor allem schwul-lesbische AktivistInnen über die Pathologisierung von Homosexualität im Diagnostic and Statistical Manual (DSM) aufgebracht, insbesondere da die Medizin damit die rechtliche Diskriminierung von Homosexuellen in den USA legitimierte. Bei den Tagungen der American Psychiatric Association (APA) gab es ab 1970 provokante Störungen. Irving Bieber beispielsweise, ein einflussreicher homophober Analytiker, wurde folgendermassen kommentiert: Wenn er in seinem Buch über schwarze Menschen so reden würde, wie er über Homosexuelle redet, dann würde er verdient gevierteilt werden.

Der Psychiater Robert L. Spitzer, der Mitte der 1970er Jahre beauftragt wurde, das DSM zu überarbeiten, war offen für wissenschaftliche Argumente zur Beseitigung der Homosexualitätskategorie aus dem DSM. Gleichzeitig verpflichtete er sich, antihomosexuellen Experten weiterhin Gehör zu schenken und organisierte ein Panel auf der APA-Tagung in Honolulu im Mai 1973, auf dem kontrastierende Meinungen vertreten wurden. Charles Socarides und Irving Bieber traten als dezidierte Gegner der Entpathologisierungsbestrebungen auf. Dieses Panel beschreibt Herzog als wichtigen Wendepunkt (2015, 422-424). Ein halbes Jahr später, im November 1973, stimmte das Kuratorium der APA dafür, die Diagnose «Homosexualität» aus ihrem Diagnoseschlüssel zu entfernen, woraufhin sich im europäischen Raum die Einschätzung der Homosexualität auch in der Psychoanalyse zu wandeln begann. ${ }^{3}$

In der Schweiz gilt Fritz Morgenthaler ${ }^{4}$ als Vorreiter dieser Entwicklung auf theoretischer Ebene, und er nahm, insbesondere durch die Mitbegründung 
des Psychoanalytischen Seminars Zürich (PSZ), auch auf institutioneller Ebene Einfluss. Er befreite die Homosexualität vom grundlegenden Stigma der Krankheit, indem er annahm, dass Sexuelles an sich, in welcher Form auch immer, niemals krankhaft sein könne. Bezüglich der Heterosexualität sei dies schliesslich auch nie vermutet worden (vgl. Morgenthaler, 2004).

In Deutschland dagegen blieb eine Diskussion fast völlig aus (vgl. Ermann, 2013, S. 32). Dort führte die Bundesarbeitsgemeinschaft Schwule im Gesundheitswesen (BASG) eine Untersuchung in Form einer deutschlandweiten Befragung psychoanalytischer Institute bezüglich ihrer Zulassungspraxis für homosexuelle KandidatInnen durch. Die Ergebnisse sind 1985 in einem Artikel in der Psyche diskutiert worden, beginnend mit der Auskunft einer bekannten Psychoanalytikerin:

Zwar gäbe es keine vernünftige theoretische oder praktische Rechtfertigung, Homosexuelle allein aufgrund ihrer Homosexualität von der Ausbildung zum Psychoanalytiker auszuschließen, dessen ungeachtet aber sei gerade dies die gängige Praxis der psychoanalytischen Institute in der Bundesrepublik, [...] «Gott sei Dank bewerben sich ja nicht so viele». (BASG, 1985, S. 553)

Von den 14 Instituten, die auf die Befragung geantwortet haben, lehnte nur das Düsseldorfer Institut die Aufnahme homosexueller BewerberInnen explizit ab: «Homosexuelle nehmen wir an unserem Institut nicht an.» (ebd., S. 554/560) In den meisten anderen Antworten auf die Frage, ob Homosexuelle zur Ausbildung zugelassen würden, zeigte sich eine deutliche Ambivalenz, die sich in der Formulierung zusammenfassen lässt: «Im Prinzip ja, aber ...» Rauchfleisch kritisiert:

Wann immer man die allgemeinen theoretischen Erwägungen mit der Praxis konfrontiert, zeigt sich das gleiche blamable Bild: ein höchst verunsichertes Verhalten der Entscheidungsträger, Rückfragen bei übergeordneten Gremien und schließlich zumeist Ablehnungen. Selbst wenn einige lesbische und schwule Kandidaten in einzelnen psychoanalytischen Instituten aufgenommen werden, fällt auf, dass dies quasi "unter der Hand» geschieht und nach außen möglichst verschwiegen wird. (Rauchfleisch, 2001, S. 149) 
Auch Parin vertritt die Meinung, Homosexuelle würden «schon im Vorfeld» aussortiert werden. «Wie das jeweils vor sich geht, ist nicht feststellbar» (Parin, 1985, S. 563). Zumindest eine Vorstellung davon, wie das vor sich gegangen sein könnte, soll die nun folgende Geschichte von Angela Hennig vermitteln. Zu lesen ist sie in dem Wissen um die Situation des Ausschlusses «offen» homosexueller Frauen und Männer von der psychoanalytischen Ausbildung in dieser Zeit - verbunden mit dem drängenden Wunsch, als eine in gleichgeschlechtlicher Partnerschaft lebende Psychologin Psychoanalytikerin zu werden. Ein Dilemma, mit dem ein Umgang gefunden werden musste. Laut der Bundesarbeitsgemeinschaft Schwule im Gesundheitswesen sei eine Empfehlung von Psychoanalytikern gewesen: «Lügt Euch doch durch», eine andere: «Macht Eure Ausbildung doch ausserhalb der Institute» (vgl. BASG, 1985). Angela Hennig hat jedoch ihren eigenen Weg gefunden - einen unter den damaligen Verhältnissen möglichen.

\section{Die Geschichte von Angela Hennig}

Angela Hennig hat sich bereit erklärt, ihre Ausbildungsgeschichte als Lesbe zu erzählen: «Ich bin jetzt 64 und stehe eher am Ende meiner beruflichen Laufbahn. Ich habe nichts mehr zu verlieren. Im Gegenteil habe ich das deutliche Gefühl, die Auseinandersetzung mit meiner Geschichte, auch auf diese Art, ist ein Gewinn für mich.» Aktuell ist sie als Psychoanalytikerin in einer Gemeinschaftspraxis in Berlin tätig. Interessanterweise liegt ihr Behandlungsraum separiert von allen anderen Praxisräumen und hat einen eigenen Zugang. Diese räumliche Situation zeigt sich mir als ein erstaunlich treffendes Sinnbild für ihre Geschichte, in der Hennig ebenfalls einen ganz eigenen Zugang zu ihrer Ausbildung und (klinischen) Praxis finden musste.

Hennig wuchs in Stuttgart auf. Die Psychoanalyse gehörte schon in der Kindheit zu ihrem Leben. Alle Familienmitglieder befanden sich zeitweise in Psychoanalyse, Erwachsene wie Kinder. Sie selber machte bereits als Kind eine psychoanalytische Therapie. Dazu erinnert sie sich, es mit der Abstinenz ihrer Kinderanalytikerin manchmal schwer gehabt zu haben - und doch hat sie die Analyse als sehr positiv, hilfreich und «lebensrettend in einer schwer traumatisierten Nachkriegsfamilie» in Erinnerung. So verwundert es nicht, dass sie schon früh den Berufswunsch der Psychoanalytikerin entwickelte, der sich im Laufe ihres Heranwachsens zunehmend festigte und konkretisierte. Sie studierte Psychologie an der Technischen Universität Berlin und sie verliebte sich in eine Frau. Ihr Coming-out als Lesbe bereitete ihr wenig Mühe, sie war überzeugt, den für sie richtigen Weg gefunden zu haben. Ihr war wichtig, in ihrem sozialen Umfeld das leben 
zu können, was sie empfand. Was ihr aber mit dem Herannahen der Ausbildung auffiel, war die Tatsache, dass keine lesbischen Psychoanalytikerinnen existierten, auch keine schwulen Psychoanalytiker - zumindest waren keine sichtbar und es schien keinen Weg zu geben, eine/n zu finden.

«Die frühen 1980er waren ganz anmeldestarke Semester an den Berliner psychoanalytischen Ausbildungsinstituten», erinnert sie sich. «Die Institute mussten nicht aufpassen, dass sie zu wenige Anmeldungen hatten.» Sie hätten eine Vorauswahl in Form von bestimmten Ausbildungsvoraussetzungen (Alter, berufliche Erfahrung usw.) festgelegt, die ein Interessent bereits erfüllt haben musste, um zu einem ersten Zulassungsgespräch eingeladen zu werden. In mehreren Zulassungsgesprächen, so Hennig, wurde von erfahrenen Psychoanalytikern über die psychischen Voraussetzungen des Bewerbers/der Bewerberin für eine psychoanalytische Ausbildung befunden.

Von anderen hatte Angela Hennig gehört, dass homosexuelle Menschen mit einer Ablehnung ihrer Bewerbung um einen Ausbildungsplatz an psychoanalytischen Instituten rechnen mussten - und das wohl nicht nur an IPA-anerkannten Instituten. Diese inoffizielle Information fand sie später, 1985, in dem oben erwähnten Artikel der Bundesarbeitsgemeinschaft Schwule im Gesundheitswesen in der Psyche bestätigt. Nur mit einem besonderen Aufwand war es Hennig einige Monate nach Erscheinen der Zeitschrift gelungen, mit einem der Autoren des BASG-Artikels Kontakt aufzunehmen. Ihre Hoffnung, über diesen zu erfahren, wo in Berlin oder anderswo in Deutschland es noch homosexuelle AusbildungskanditatInnen gebe, und wie sie sich mit ihnen in Verbindung setzen könnte, wurde aber enttäuscht.

Da für Hennig eine andere Berufswahl nicht vorstellbar war, hat sie 1981 begonnen, intensiv nach Möglichkeiten zu forschen, die Ausbildung trotz der zu erwartenden Schwierigkeiten absolvieren zu können: An welchen Instituten musste mit welchen Hürden und mit welchen Restriktionen zu rechnen sein? Sie erfuhr, dass es Institute gab, an denen eine Ausbildung nur unter konsequenter Verleugnung der eigenen sexuellen Orientierung möglich gewesen wäre. Das hätte für Angela Hennig bedeutet, selbst in der Lehranalyse nicht authentisch berichten zu dürfen, was in ihr vorgehe. Sie hätte die eigene Zensur nicht vollständig ausschalten können - was für sie mit dem Wunsch nach einem gelingenden analytischen Prozess kollidierte. Es wäre dann nötig gewesen, aus der eigenen homosexuellen Beziehung eine andere Beziehungsform zu machen, z. B. eine Freundschaft, oder ihrer Freundin ein anderes Geschlecht anzudichten oder sie gar ganz zu verleugnen - mit dem Risiko für nicht beziehungsfähig gehalten zu werden, oder etwas 
zu erfinden, um genau diesem Risiko entgegenzuwirken. Einige Institute galten als sehr rigide, andere schienen Homosexualität zu ignorieren.

Das Psychoanalytische Seminar Zürich (PSZ) hatte in den frühen 1980er Jahren den Ruf, dass man dort als homosexueller Mensch gut eine psychoanalytische Ausbildung machen könne. Angela Hennig hatte die Möglichkeit, sich in der Schweiz ausbilden zu lassen, in ihre Überlegungen einbezogen, sich dann aber aus privaten Gründen dagegen entschieden. Sie wählte unter den Berliner Instituten dasjenige aus, bei dem sie ihren Recherchen entsprechend am ehesten die Chance haben würde, die Ausbildung zur Psychoanalytikerin absolvieren zu können.

Heute sagt sie, dass ihr damals diese Ausbildung so wichtig gewesen sei, dass sie kaum Gedanken daran verschwendet habe, welchen Preis sie dafür zu zahlen hätte. Im Nachhinein denkt sie, das war auch gut so, denn sonst wäre sie in diesem Prozess noch zusätzlich innerlich behindert worden.

Nach dem, was Hennig unter der Hand erfahren konnte, war eine Zulassung zur Ausbildung an dem von ihr ausgewählten Institut nur dann möglich, wenn es ihr gelingen würde, in den Zulassungsgesprächen eine Abweichung von der erwünschten (also hetero-) sexuellen Orientierung nicht zur Sprache zu bringen und dieses Detail bei direkter Nachfrage möglichst zu umgehen. Diese Information hatte offenbar schon viele InteressentInnen dazu bewogen, sich nicht zu bewerben. Angela Hennig aber konnte es mit sich vereinbaren, sich auf diese Weise den «Zutritt» ins Institut zu verschaffen, sollte das die einzige Möglichkeit sein. Wichtiger war ihr die Lehranalyse. Dort wollte sie authentisch sein können. Sie hatte erfahren, dass es dann möglich sei, die eigene von der Hetero-Norm abweichende sexuelle Orientierung in der Lehranalyse zu thematisieren, wenn man eine/n entsprechende/n Lehranalytiker/in fand, der/die das mit trug. Diese/r Lehranalytiker/in musste demnach bereit sein, Hennigs sexuelle Orientierung vor den KollegInnen zu verschweigen.

Endlich fand ein erstes Zulassungsgespräch statt, von dem sich Angela Hennig allerdings noch keine direkte Aufnahme erhoffte, da sie die Aufnahmebedingung der beruflichen Vorerfahrung noch nicht komplett erfüllte. Dieses Gespräch habe ausgerechnet drei Tage nach der Trennung von ihrer ersten Freundin stattgefunden. In diesem Zustand habe sie ihre Abwehr als «sehr löchrig» empfunden, weshalb sie sich fest vorgenommen habe, über alles andere zu reden als über Beziehung. Das sei ihr auch gelungen. Das Gespräch habe bei einem alten Kollegen stattgefunden, einem Jungianer ${ }^{5}$. Sie selbst wollte die freudianische Richtung einschlagen. Die erwartete Ablehnung kam. Ein Jahr später habe sie eine Wiedereinladung erhalten. Diesmal habe das Gespräch mit einem älteren Freudianer stattgefunden. Wieder 
habe sie sich intensiv vorbereitet. Das Thema «Liebesbeziehung» liess sich nicht mehr umgehen. Nach offen gebliebenen Themen gefragt, hielt sie sich allgemein, indem sie erklärte, ihre Beziehung sei damals bewusst nicht von ihr thematisiert worden, da diese gerade drei Tage vor jenem Gespräch beendet worden sei. In der Annahme, es handele sich um eine Beziehung zu einem Mann, habe der Analytiker nach dem Grund für die Trennung von «ihm» gefragt. Im Anschluss hätte sie über diese Beziehung sprechen können, ohne die Partnerin als weiblich kennzeichnen zu müssen. Eine Korrektur der falschen Annahme hätte möglicherweise zu einer abschlägigen Beurteilung geführt, vermutlich mit einer völlig anderen Begründung. Dies jedenfalls hatte Hennig damals aus den gehörten Geschichten ableiten können. Nach einem positiven Gesprächsverlauf sei sie ein drittes Mal eingeladen worden. Das dritte Gespräch führte sie mit einer Analytikerin, von der sie sich mit ihrer beruflichen Situation, die gerade nicht ganz einfach war und im Zentrum des Gesprächs stand, gut verstanden fühlte.

Schliesslich sei befunden worden, Angela Hennig erfülle alle Voraussetzungen, die psychoanalytische Weiterbildung beginnen zu können. Mit dem Schritt der LehranalytikerInnen-Suche hatte sie sich bereits lange vor ihrer Zulassung zur Ausbildung befasst. Da sie bereits eine therapeutische Analyse bei einer Frau gemacht habe, erwog sie nun, die Lehranalyse bei einem männlichen Analytiker zu absolvieren, nicht zuletzt, um nicht den Verdacht zu wecken, sie sei lesbisch. Für das Vorgespräch mit ihrem zukünftigen Lehranalytiker hatte sie sich fest vorgenommen, offen zu sagen, dass sie lesbisch ist, und auf dessen Einhaltung der Schweigepflicht zu vertrauen. Nach einem entsprechend offenen Gespräch hatten sich beide auf die Durchführung einer Lehranalyse nach der Zulassung von Angela Hennig zur Ausbildung geeinigt. Allerdings gab dieser Analytiker zu bedenken, dass sie ja keine heterosexuellen Männer behandeln könne. Kurz nach ihrer Zulassung zur Ausbildung erteilte er ihr eine Absage. Eine Begründung erhielt sie nicht.

Hennig blieb standhaft und wandte sich in einem weiteren Versuch an die Psychoanalytikerin, bei der sie das dritte, gut verlaufene Zulassungsgespräch gehabt hatte. In dem Vorgespräch für eine Lehranalyse bei ihr sei eine Einigung so gut wie erfolgt gewesen, bis Hennig auch hier ihre sexuelle Orientierung bekannt gab. Als Antwort bekam sie zu hören: «Solche wie Sie wollen wir hier nicht.» Die Analytikerin kündigte prompt an, dass sie dies dem Unterrichtsausschuss melden und beantragen werde, dass Hennigs Zulassung zur Ausbildung zurückgezogen werde. 
Hennig hielt an ihrem Vorhaben fest, einen Lehranalytiker zu finden. Es habe da eine Analytikerin gegeben, zu der sie sehr gerne in die Lehranalyse gegangen wäre. Ihre inzwischen allzu grosse Verunsicherung habe sie jedoch davon abgehalten, diese anzufragen. Sie sehnte sich nach Absicherung. Aufgrund der Informationen, die sie über einen männlichen Analytiker erhalten konnte, entschied sie sich für ein Vorgespräch bei ihm. Diesmal platzte sie gleich zu Beginn des Gesprächs heraus: «Ich bin lesbisch. Nehmen Sie mich trotzdem?» Nach dessen Zusage begann endlich die Lehranalyse. Als Hennig eine schriftliche Vorladung vom Unterrichtsausschuss erhielt, konnte sie gemeinsam mit ihrem Lehranalytiker besprechen, wie mit dem Antrag der Analytikerin, die Zulassung zur Ausbildung zurückzuziehen, umzugehen sei. Er versprach, das intern zu regeln. Ob er das tatsächlich getan hat, hat Hennig nie erfahren. Dessen ungeachtet habe er ihr noch die beiden Ausschlusskriterien für eine psychoanalytische Ausbildung genannt: Psychose und Kriminalität. Sie solle doch mal diese beiden Dinge verneinen und dann abwarten, was passiere.

Das Gespräch mit der Vorsitzenden des Unterrichtsausschusses fand statt - darin teilte diese Angela Hennig zunächst mit, es sei empfohlen worden, ihre Zulassung zurückzuziehen, und fragte, ob sie sich Gründe dafür vorstellen könne. Wie vom Lehranalytiker empfohlen, nannte Hennig zunächst die beiden Ausschlusskriterien und versicherte, beides könne es nicht sein. Auf die Frage, was es sonst sein könne, sagte Hennig: «Meine Frauenbeziehung.» Als Antwort wünschte die Vorsitzende des Unterrichtsausschusses ihr viel Erfolg bei der Ausbildung. Als Angela Hennig den Gesprächsraum verliess, kamen ihr die Tränen. Heute sagt sie: «Das hat die Frau tun müssen. Das war ihre Aufgabe.»

Zur Aufnahmesituation sagt sie heute: «Das sind Einzelentscheidungen der Psychoanalytiker gewesen, die die Zulassungsgespräche geführt haben.» Sie hat sich Jahre später ihre Akte kommen lassen, aus der hervorging, dass sie in allen drei Zulassungsinterviews sehr gute Voten bekommen hatte. Die Analytikerin, die das dritte Zulassungsgespräch mit ihr geführt hatte, hatte in einem Brief an den Unterrichtsausschuss geschrieben, es hätten sich neue Gründe ergeben, weshalb die Zulassung zurückgezogen werden müsse. Hennigs sexuelle Orientierung war an keiner Stelle in der Akte vermerkt.

Zumindest schien nun der Ausbildung nichts mehr im Wege zu stehen, wobei Angela Hennig deutlich spürte, auf welch wackligem Boden sie sich befand. Völlig klar sei ihr gewesen, dass sie niemandem am Institut von ihrer sexuellen Orientierung berichten dürfe, wenn sie die Ausbildung erfolgreich beenden wolle. Sie wusste auch, dass sie darauf vertrauen musste, dass ihr Lehranalytiker nieman- 
dem davon berichte. Dies habe sie in ein besonders unangenehmes Abhängigkeitsverhältnis zu ihm gebracht, da er ja seine Zustimmung zum Abschlussexamen geben musste oder diese eben verweigern konnte - was auch mehrfach auf die Probe gestellt worden sei. Beispielsweise hatte sich im Verlauf der Ausbildung eine sehr gute Freundschaft mit einer anderen Ausbildungskandidatin entwickelt und Hennig gab irgendwann ihrem Bedürfnis nach, dieser Freundin von ihrer Liebesbeziehung zu einer Frau zu erzählen. Das wurde von der neuen Freundin auch gut aufgenommen. Hennig hatte jedoch nicht gewusst, dass deren Ehemann bei Hennigs Lehranalytiker ebenfalls eine Lehranalyse machte und in dem Rahmen auch von ihr und ihrer lesbischen Beziehung berichtete. In der darauffolgenden Lehranalysestunde wies ihr Lehranalytiker sie zurecht: «Wenn Sie das so frei herumerzählen, dann können Sie das mit ihrem Ausbildungsabschluss vergessen».

Hennig engagierte sich schon zu Beginn der Ausbildung sehr intensiv am Institut. Beispielsweise liess sie sich in den Studentenausschuss wählen. Ein solches institutspolitisches Engagement habe sie später bei anderen lesbischen und schwulen Ausbildungskandidaten ebenfalls beobachtet. Es habe sich aus einem Schutzbedürfnis heraus entwickelt, quasi aus dem Wunsch, hinter die Kulissen zu schauen, um sich weniger ausgeliefert zu fühlen. Zudem habe sie mit ihrem Engagement zeigen wollen, dass sie etwas könne und die Zugehörigkeit verdiene.

Der Frauentherapiekongress war ein wichtiger Ort für Hennig in der Auseinandersetzung mit anderen feministischen und auch lesbischen Therapeutinnen. Dort hat sie sich wesentlich wohler gefühlt als am eigenen Ausbildungsinstitut. Sie hat andere lesbische Therapeutinnen kennen gelernt und Gelegenheit gehabt, sich mit ihnen auszutauschen. Einfach war auch das nicht immer, da sie sich dort zwar als lesbische Therapeutin zu Hause fühlen konnte, die Psychoanalyse aber galt besonders bei den Feministinnen als frauenfeindlich, sodass sie auch dort nicht voll akzeptiert wurde.

Schliesslich gründete Hennig mit Psychoanalytikerinnen und Ausbildungskandidatinnen aus der Bundesrepublik Deutschland und der Schweiz, die sie dort kennen gelernt hatte, eine «lesbische Psychoanalytikerinnengruppe». Sie trafen sich von 1987-1991 etwa drei bis viermal jährlich übers Wochenende und richteten die Treffen reihum aus. Diese Gruppe gab ihr Ruhe und Halt. Im Grunde sei sie ihre Rettung gewesen. Dort konnte sie reden und dort wurde sie gehört. Sie konnte sich zeigen, wie sie war, und fand Austausch mit Kolleginnen, die sich in ähnlichen Situationen befanden. Der Austausch wurde von allen Gruppenteilnehmerinnen als sehr entlastend erlebt. Das Zusammengehörigkeitsgefühl in der Gruppe wirkte dem sonst so präsenten Vereinzelungsgefühl in der jeweiligen Stadt, am jeweiligen 
Institut entgegen. Klar war, dass dieser Zirkel zum Schutz aller Beteiligten inoffiziell bleiben musste. Inhaltlich ging es um Ausbildungsfragen, um die Selbsterfahrung, und auch Intervision fand statt. Literatur wurde diskutiert, beispielsweise von Jessica Benjamin, Eva S. Poluda Korte, Nancy Chodorow, Joyce McDougall und Christa Rhode-Dachser. Eigene Publikationen und Vorträge wurden besprochen.

Auf dem Frauentherapiekongress hatte Angela Hennig öffentlich gemacht, dass sie eine lesbische Psychoanalytikerin und feministisch sei. Dadurch und auch über die verschiedenen Frauentherapieprojekte, bei denen sie auf Listen geführt wurde, hat sie über viele Jahre einen grossen Zulauf an Patientinnen gehabt. Sie sei lange die einzige Psychoanalytikerin in Berlin gewesen, von der man gewusst habe, dass sie lesbisch sei, oder es zumindest hatte herausfinden können, wenn man wollte. Bis in die Mitte der 1990er Jahre sei sie von mindestens zehn Psychologinnen und Ärztinnen gefragt worden, wie man als Lesbe eine psychoanalytische Ausbildung machen könne. Das habe ihr das Elend sehr deutlich gemacht. Die letzte Frau, von der sie angefragt worden sei, sei die erste gewesen, die die Ausbildung offiziell als Lesbe gemacht habe - das heisst, dass sie in keinem Bereich ihre eigene sexuelle Orientierung habe verleugnen müssen: Im Zulassungsgespräch, in ihrer Lehranalyse und vor anderen Ausbildungskandidatinnen habe diese Kollegin offen über ihre lesbische Orientierung reden können.

Hennig blickt zurück: «Die ganze Zeit lang habe ich mir gesagt: Wenn Du als Lesbe Psychoanalytikerin werden willst, dann musst Du da durch.» Verständnis war nur in den seltenen Gruppentreffen zu erwarten. Nach dem Examen konzentrierte sie sich voll auf ihre Arbeit als Psychoanalytikerin und wollte mit dem Thema "Auseinandersetzung mit dem Institut» nichts mehr zu tun haben. Acht Jahre nach dem Examen ist Hennig dann konsequenterweise aus dem Institut ausgetreten. Der Begriff «Pseudoheimat» fällt ihr ein. Ihr Gefühl beschreibt sie mit den Worten: «Aus dem Nest gestossen, wo nie ein Nest war.» Durch ihren wortlosen Austritt habe sie aber auch keine gute Ablösephase gehabt. «Ich bin einfach gegangen. Kein Schimpfen, keine Idealisierung, ich habe nicht gesprochen. ... wie ausgeblendet. Inzwischen habe ich Lust auf eine Wiederannäherung. Unklar ist, ob mir das gelingt. In jedem Fall wünsche ich mir irgendeine Zugehörigkeit.» Bis zum Examen hatte sie im Institut wenige Kontakte zu anderen KandidatInnen. Sie spürte deutlich, dass sie sich nicht traute, sich zu öffnen.

Während der Lehranalyse hatte sich Hennig neu verliebt, doch schien es nicht möglich zu sein, das zu thematisieren. Ein freies Assoziieren blieb zu ihrem eigenen Erstaunen und entgegen ihrer Erwartung blockiert. Als der Lehranalytiker in einer konflikthaften Situation am Ende der Lehranalyse ärgerlich bemerkte, 
dass die Analyse ja nichts genützt habe, sie sei ja noch immer lesbisch, habe sie ihre Hemmung verstanden.

In verschiedenen Zusammenhängen wurden Hennig als Reaktion auf die Bekanntgabe ihrer sexuellen Orientierung Fragen gestellt, die Zweifel an ihrer Fähigkeit ausdrückten, bestimmte Personengruppen zu behandeln. Jemand fragte, wie sie sich denn vorstelle, Männer zu therapieren. Der Nächste stellte die gleiche Frage in Bezug auf schwule Männer, ein anderer fragte, wie sie Frauen behandeln wolle. Schliesslich verdeutlichte ihr die Widersprüchlichkeit dieser Fragen untereinander, dass es sich hier um persönliche Vorurteile und nicht um wissenschaftlich begründete Zweifel handeln musste.

\section{Schluss}

Diese Vorurteile implizieren die Frage, ob Homosexuelle überhaupt Psychoanalytiker sein können. Ich bin mit Ermann der Meinung, dass derartige Fragen als überflüssig betrachtet werden können, weil sie vom Ideal des absoluten Verstehens ausgehen, das keine Grenzen kennt. Entscheidender ist die Frage, ob AnalytikerIn und AnalysandIn es aushalten können, dass es in dieser besonderen Begegnung wie der Psychoanalyse einen Rest an Differenz gibt, der anerkannt aber nicht beseitigt werden kann. Zu bedenken bleibt die Grundtatsache, die schon Freud seinen Anhängern vermitteln wollte: Die sexuelle Orientierung ist nur einer unter den konstitutiven Faktoren unserer Beziehungen. Es geht immer um den Blick auf die Gesamtpersönlichkeit (vgl. Ermann, 2013, S. 46-47).

Nun sind hinsichtlich der Zulassungsbedingungen für Homosexuelle an den Instituten seit den 1980er Jahren Entwicklungsprozesse zu verzeichnen. Robert J. Stoller trug schon an der APA-Tagung in Honolulu 1973 mit aussergewöhnlichem Nachdruck die kreativste Kritik «gegen die herrschende heterosexuelle männliche Norm, spöttisch und tiefernst zugleich» vor (Herzog, 2015, S. 427). Rauchfleisch zitiert Stoller, der 1983 die Missstände in den psychoanalytischen Ausbildungsinstituten und in der psychoanalytischen Theorie generell aufgriff:

Wir haben aus Diagnosen Anklagen gemacht und dabei unser Verhalten mit Fachjargon kaschiert. Aber obwohl dieser den Hass verdeckt, verstärkt er die Grausamkeit; der Jargon ist das Urteil. (Rauchfleisch, 2001, S. 147)

Erst 1991, 18 Jahre nachdem die «Psychiatrie» sich offiziell von der Diagnose «Homosexualität» distanziert hatte, verabschiedete die APV als erste psychoanaly- 
tische Fachgesellschaft eine Antidiskriminierungsklausel, die die Ablehnung von BewerberInnen allein aufgrund ihrer sexuellen Orientierung untersagte. So bekamen die ersten offen homosexuellen Menschen Zugang zur psychoanalytischen Ausbildung.

Der Wandel kam nach vielen Jahren sorgfältiger Verhandlungen. Ein entscheidender Faktor, der diese Entwicklung ermöglichte, war die «Feminismus-isierung der Psychoanalyse», die aus der breiteren feministischen Revolution in allen Zweigen der Psychotherapie im Laufe der 1980er Jahre entstand. (Herzog, 2015, S. 431)

Im ICD-10 wurde der Beschluss der Weltgesundheitsorganisation (WHO) vom 17. Mai 1990, Homosexualität aus ihrem Krankheitskatalog zu streichen, umgesetzt. ${ }^{6}$

1992 befragte Rauchfleisch psychoanalytische Ausbildungsinstitute im deutschsprachigen Raum danach, «welche Haltung sie derzeit bezüglich der Zulassung von Kandidaten einnehmen, welche sich offen zu ihrer Homosexualität bekennen» (2001, S. 149). Fünf der 34 antwortenden Institute berichteten von bereits erfolgten Aufnahmen von Lesben und Schwulen, zwei kritisierten die traditionelle restriktive Aufnahmepraxis in derVergangenheit, ein Institut sprach sich gegen die Aufnahme Homosexueller aus. Die meisten Antworten zeigten «eine Ambivalenz zwischen einem offenen «Nein` und dem Wunsch, diese Ablehnung durch Rückgriff auf psychoanalytische Theorien zu rationalisieren» (Rauchfleisch, 2001, S. 150). 1993 plädierte Rauchfleisch dafür,

... gerade am Thema der Homosexualität zu zeigen, dass die Psychoanalyse nach wie vor ein grosses emanzipatorisches Potenzial in sich birgt, mit dessen Hilfe wir irrationale Vorurteile und Ausgrenzungsmechanismen aufdecken und auflösen können. (Rauchfleisch 1993)

Diese Aussage unterstütze ich vollständig und halte sie noch heute für gleichermassen gültig.

Das sich nur langsam verändernde Entwicklungsbild spiegelte sich beispielsweise in Zeitungsartikeln folgendermassen wider: 
In der Regel werden Homosexuelle, aus mehr oder weniger offensichtlichen Gründen, nicht zur Psychoanalyseausbildung zugelassen. Nur wenigen gelingt es, an liberaleren Instituten, zum Beispiel in Frankfurt oder München, unterzukommen. Die Berufsdiskriminierung in diesem Sektor ist ein offenes Geheimnis. (Roedig, 1998)

Die Internationale Psychoanalytische Vereinigung (IPV) folgte der APV 2002 mit einer ähnlichen Stellungnahme. Dennoch blieb auch in den Jahren danach die Situation für Lesben und Schwule, die sich für die psychoanalytische Ausbildung interessierten, unübersichtlich: An welchen Instituten würden sie mit welchen Begründungen angenommen oder auch abgelehnt werden, wenn sie ihre Homosexualität offen äussern würden? (Homepage des DPG-Instituts Hamburg) ${ }^{7}$ Trotz der Entwicklung von anerkannten nicht-pathologisierenden Theorien im deutschsprachigen Raum änderte sich an der faktischen Diskriminierung homosexueller KollegInnen kaum etwas.

Einen Grund für den Unterschied zur nordamerikanischen Situation, wo lesbische und schwule AnalytikerInnen auf allen Ebenen so selbstverständlich geworden sind, dass sie niemandem mehr der Rede wert sind, sieht Quindeau (2015, S. 651) im amerikanischen Rechtssystem, das von öffentlichen Institutionen eine aktive Nicht-Diskriminierungspolitik verlangt. Das Positionspapier der APA "Position Statement on Homosexuality and Civil Rights" (1973) verurteilte die Diskriminierung Homosexueller und forderte die Regierung auf, Homosexuellen denselben rechtlichen Schutz zukommen zu lassen wie anderen Minderheiten. 2015 stellte Quindeau fest:

Die Haltungen zur Homosexualität oder-weiter gefasst-zu LGBT-
Fragen sind in den psychoanalytischen Gesellschaften der IPA sehr
unterschiedlich ausgeprägt. Während in Nordamerika bereits die
ersten transgender Kandidat_innen ausgebildet werden, tun sich
Institute in Deutschland und anderen europäischen Ländern noch
schwer mit homosexuellen Bewerber_innen. (Quindeau, 2015,
S. 649)

Obwohl die Diskriminierung von Homosexualität inzwischen kritisiert und weiterführende Theorien entwickelt worden sind, setzen sich Ausgrenzung und Diskriminierung fort. Analytikerinnen und Analytiker gehören Institutionen an, 
die selbst massiv ausgegrenzt haben. Heute noch kommen diese Ausgrenzungen vor, wohl meist unabsichtlich (vgl. Quindeau, 2015, S. 653). Unter Bezugnahme auf den eingangs erwähnten Filmtitel ${ }^{8}$ könnte man hier feststellen: «Nicht die Homosexuellen, die sich an den Instituten bewerben, sind problematisch, sondern bestimmte Verhältnisse, die sie dort vorfinden.» Welche Verhältnisse wären aber wünschenswert?

Bereits 1985 plädierte Parin dafür, dass für die Zulassung homosexueller KanditatInnen dieselben Kriterien gelten müssten wie für jede andere Kandidatur. Als hinderlich dafür befand er die Tendenz der Ausbildungsinstitute, sich der jeweils herrschenden Ideologie anzupassen. Tatsächlich versuchten die Institute noch Anfang der 1980er Jahre dehnbar formulierte psychologische Merkmale mittels bestimmter Interview-Methoden festzustellen, was nach Parin die Auswirkung bewusster und unbewusster Vorurteile gegen Homosexuelle zulasse, «solange im betreffenden Institut kein prinzipielles Einverständnis über die Unzulässigkeit einer solchen Vorurteilsbildung besteht» (1985, S. 563). Diesem Vorschlag folgend halte ich eine vertiefte Reflexion derVorurteilsbildung an jedem einzelnen Institut für sinnvoll. Ebenso eine weitere theoretische Auseinandersetzung mit Themen wie der Entwicklung von verschiedenen Formen von Sexualität.

Parin kritisierte, dass das Auswahlverfahren vor Beginn der Ausbildung Qualitäten von Bewerbern prüfe, die in der Hauptsache erst nach der Ausbildung beurteilt werden könnten. Es «erstellt also einen «numerus clausus», der auf obligat subjektiven, prognostischen Überlegungen und Annahmen beruht» (Parin, 1985, S. 563). Dieser Kritik entsprechend sollten die bestehenden Auswahlverfahren überprüft werden.

Würde man die verdeckende Ausbildungssituation insgesamt klären, müsste man nicht mehr sagen: «Gott sei Dank bewerben sich ja nicht so viele». Die Frage ist also, wie wir Ausbildungsbedingungen schaffen können, die nicht diskriminieren, sodass wir nicht erst in die Situation kommen, befürchten zu müssen, dass sich jemand bewirbt.

\section{Literatur}

BASG (Bundesarbeitsgemeinschaft Schwule im Gesundheitswesen). (1985). Psychoanalyse in Schwulitäten. PSYCHE - Z Psychoanal 39, 553-560.

DPG-Institut Hamburg. (2016). Institut für Psychoanalyse und Psychotherapie e.V. der Deutschen Psychoanalytischen Gesellschaft. http://www.dpg-instituthamburg.de/index.php/selbstverstaendnis?id=141 
Ermann, M. (2013). Die Entwicklung der normalen männlichen Homosexualität. Vortrag am Daseinsanalytischen Seminar DaS Zürich, 26. Januar 2013. In: Bulletin 2013 der European Federation for Psychoanalytic Psychotherapy Schweiz. Psychoanalyse und sexuelle Orientierung.

Freud, S. (1905). Drei Abhandlungen zur Sexualtheorie. Gesammelte Werke. Band V. 27145. Frankfurt a. M.: S. Fischer.

Herzog, D. (2015). Die bemerkenswerte Beständigkeit der Homophobie in der Psychoanalyse. Analytische Psychologie. Zeitschrift für Psychotherapie und Psychoanalyse. Heft 182, 414-437.

Kreische, R. (2005). Homosexualität: Angst vor Fremdem. PP 43. Ausgabe März 2005.

Morgenthaler, F. (2004). Homosexualität, Heterosexualität, Perversion. Giessen: Psychosozial-Verlag.

Parin, P. (1985). Kommentar zu «Psychoanalyse in Schwulitäten» von der Bundesarbeitsgemeinschaft Schwule im Gesundheitswesen. PSYCHE-ZPsychoanal 39, 561-564.

Quindeau, I. (2015). «Recovering from Iatrogenenesis ...». Vom Umgang mit dem homophoben Erbe. PSYCHE - Z Psychoanal 69, 648-660.

Rauchfleisch, U. (1993). Homosexualität und psychoanalytische Ausbildung. Forum Psychoanal. 9, 339-347.

Rauchfleisch, U. (2001). Schwule, Lesben, Bisexuelle. Lebensweisen - VorurteileEinsichten. Göttingen: Vandenhoeck \& Ruprecht.

Roedig, A. (29.12.1998). Ödipus, anders gelöst. Für die Psychoanalyse sind Homosexuelle nach wie vor gestört. Tagesspiegel.

Schigl, B. (2012). Psychotherapie und Gender. Konzepte. Forschung. Praxis. Welche Rolle spielt die Geschlechtszugehörigkeit im therapeutischen Prozess? Wiesbaden: Springer VS.

\section{Anmerkungen}

1 In Anlehnung an Quindeau (2015) unterscheide ich zwei Ebenen der Ausgrenzung: Eine konzeptionelle Ebene in den Bereichen der Theoriebildung und der klinischen Praxis sowie eine institutionelle Ebene (S. 653). Die hier beschriebene Geschichte der Berliner Psychoanalytikerin Angela Hennig steht exemplarisch für das schwierige Verhältnis der institutionellen Psychoanalyse in Deutschland zur Homosexualität.

2 Zu den neuen Entwicklungen zählte auch der in Deutschland seit 1979 jährlich stattfindende Christopher Street Day (CSD), zur Erinnerung an den Stonewall-Aufstand von 1969. Anfang der 1990er Jahre stiegen die Teilnehmerzahlen an den bundesdeutschen CSDs massiv an - in Berlin auf etwa 500000 Personen -, was nochmals einen Hinweis auf das Ausmass des damals bestandenen Wunsches Homosexueller, gesehen zu werden, gibt. 
3 Die Weltgesundheitsorganisation (WHO) dagegen führte in ihrer bis 1992 gültigen neunten Ausgabe der International Classification of Diseases (ICD) Homosexualität noch als eigene Krankheit. In der folgenden Version ICD-10 tauchte die gleichgeschlechtliche Neigung nicht mehr auf.

$4 \quad$ Fritz Morgenthaler (Schweizer Psychoanalytiker 1919-1984) war zusammen mit Paul Parin und Goldy Parin-Matthèy neben Georges Devereux einer der Begründer der Ethnopsychoanalyse und ein Mitbegründer des Psychoanalytischen Seminars Zürich (PSZ). Morgenthaler ging entgegen vieler Theorien, die Homosexualität als einen Defekt begreifen, der behoben werden muss, den gesellschaftlichen und theoretischen Missverständnissen nach, die zur Pathologisierung der Homosexualität und Perversion geführt hatten.

$5 \quad$ Innerhalb des Instituts waren verschiedene Richtungen vertreten.

6 Seit 1948 hatte dieWHO in der ICD Krankheiten erfasst und ihnen Codes zugewiesen. Die Verschlüsselung diente dazu, Diagnosen international zu deklarieren. Homosexualität war unter der Nummer 302.0 als eine psychische Krankheit aufgeführt, die man heilen könne.

7 Andere Entwicklungen im anglophonen Sprachraum: Charles W. Socarides (amerikanischer Psychoanalytiker 1922-2005), der Homosexualität zeitlebens als schwere psychische Störung ansah, war 1992 einer der Mitgründer der National Association for Research and Therapy of Homosexuality (NARTH), die für reparative Therapien eintritt (anders als die übrigen Psychotherapieverbände halten sie solche Therapien für wirksam). Dem steht der Ansatz der Gay Affirmative Psychotherapy entgegen.

8 Dokumentarfilm von Rosa von Praunheim: «Nicht der Homosexuelle ist pervers, sondern die Situation, in der er lebt».

\section{Angaben zur Autorin}

Julia Braun, Dipl. Psych., 1969, Psychoanalytikerin und Supervisorin in eigener Praxis in Zürich. Lehrtätigkeit am Psychoanalytischen Seminar Zürich (PSZ) und der Zürcher Hochschule für Angewandte Wissenschaften (ZHAW). Bis 2011 Leitende Psychologin in einem Rehabilitationszentrum für junge Erwachsene mit Schizophrenie oder Persönlichkeitsstörung. Gründungsmitglied des Dachverbandes Deutschsprachiger PsychosenPsychotherapie (DDPP). 